\title{
Neutron Calibration Sources in the Daya Bay Experiment
}

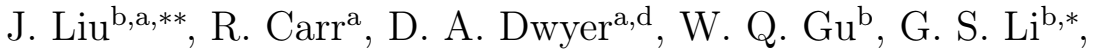

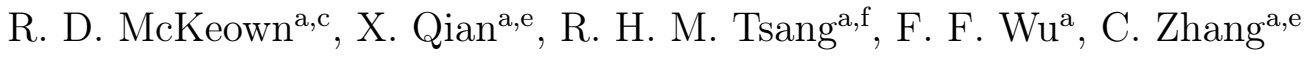 \\ ${ }^{a}$ Kellogg Radiation Laboratory, California Institute of Technology, Pasadena, California, \\ USA \\ ${ }^{b}$ Department of Physics, Shanghai Jiao Tong University, Shanghai, China \\ ${ }^{c}$ Department of Physics, College of William and Mary, Williamsburg, Virginia, USA \\ ${ }^{d}$ Lawrence Berkeley National Laboratory, Berkeley, California, USA \\ ${ }^{e}$ Brookhaven National Laboratory, Upton, New York, USA \\ ${ }^{f}$ Department of Physics and Astronomy, University of Alabama, Tuscaloosa, Alabama \\ 35487, USA
}

\begin{abstract}
We describe the design and construction of the low rate neutron calibration sources used in the Daya Bay Reactor Anti-neutrino Experiment. Such sources are free of correlated gamma-neutron emission, which is essential in minimizing induced background in the anti-neutrino detector. The design characteristics have been validated in the Daya Bay anti-neutrino detector.
\end{abstract}

Keywords:

neutron sources, ${ }^{241} \mathrm{Am}-{ }^{13} \mathrm{C}$, reactor neutrinos, $\theta_{13}$, Daya Bay, calibration

\section{Introduction}

Neutron sources are important calibration sources with a wide range of 3 applications. In modern reactor neutrino experiments such as Daya Bay [1], 4 Double Chooze [2] and RENO [3], the electron anti-neutrinos are detected 5 by liquid scintillator detectors via the inverse beta decay (IBD) reaction ${ }_{6} \quad \overline{\nu_{e}}+p \rightarrow e^{+}+n$ with the time-correlated prompt positron signal ranging 7 from 1 to $10 \mathrm{MeV}$, and the delayed neutron capture signal of $\sim 8 \mathrm{MeV}$ on the

*lgs1029@sjtu.edu.cn

**jianglai.liu@sjtu.edu.cn 
gadolinium dopant or $2.2 \mathrm{MeV}$ on hydrogen. In the Daya Bay experiment, which is located in South China, the regular deployment of neutron sources allows a thorough characterization of the detector response to IBD neutrons, which contributes to the recent discovery of the neutrino mixing angle $\theta_{13}$ [4].

The automated calibration units (ACUs) of the Daya Bay anti-neutrino detectors (ADs) are detailed in [7]. Each AD is submerged in a water pool and is equipped with three ACUs on top. Neutrino interactions are rare, so minimizing potential background created by the neutron sources is a top consideration. In this article, we discuss the design and construction of low rate $(\sim<1 \mathrm{~Hz})$ neutron sources that are free of correlated gamma emission. Although used in a specific experiment, such a source could also be potentially useful in other occasions where ultra low background is desired, or where one seeks a neutron source with no associated gamma rays.

\section{Physical requirements to the neutron sources}

As discussed in [4], the Daya Bay ADs are arranged in a 4-near and 4-far configuration to the nuclear reactor cores, with the near detectors sampling the reactor neutrino flux and far detectors detecting the $\overline{\nu_{e}}$ disappearance due to $\theta_{13}$. The rates of the IBD in the near (far) detectors are approximately 700 (70)/day/AD [4]. For each AD module, three ACUs are instrumented, each of which is capable of deploying radioactive sources vertically into the detector. There is one neutron source in each ACU [1][7]. During normal neutrino data taking, the neutron sources are "parked" inside the ACUs right above the AD. Although these neutrons rarely leak directly into the neutrino target (gadolinium loaded liquid scintillator), they can get captured on surrounding materials, in particular, stainless steel, i.e. $\mathrm{Fe}, \mathrm{Mn}, \mathrm{Cr}, \mathrm{Ni}$, etc, and emit gamma rays ranging from 6 to $10 \mathrm{MeV}$. These high energy gamma rays, later referred to as SS-capture gammas, are difficult to shield, leading to two kinds of background due to accidental and correlated coincidences: a) the SS-capture gammas can be in random delayed coincidence with ambient gamma background, mimicking the IBD signals, and b) if multiple neutrons are emitted per decay, or a neutron is emitted with a correlated gamma ray, or a neutron is producing gammas via inelastic scattering, they can form real correlated background to the IBDs. The signal to background ratio at the far site drives the requirements to the neutron sources:

- the accidental background to be less than $5 \%$ of the IBD signal at the far site. Such a background can be statistically subtracted; 
- the correlated background to be less than $0.5 \%$ of the IBD signal at the far site, i.e. $<0.35$ per day. Such a background can be estimated via Monte Carlo (MC) simulation and benchmarked by special control data.

For a typical neutron source inside the ACU, a GEANT4 [8] simulation with realistic detector geometry predicts that the SS-capture gamma ray leaking into the detector satisfying the IBD delay energy cut is approximately $2 \times 10^{-3}$ per neutron. Taking into account the $\sim 70 \mathrm{~Hz}$ singles rate [4] and $200 \mu$ s coincidence window, the first requirement translates into a limit of the neutron rate per source $<1 \mathrm{~Hz}$.

\section{Design of the neutron source}

\subsection{Selection of neutron source}

There are several types of commonly used compact neutron calibration sources, e.g. fission sources such as ${ }^{252} \mathrm{Cf},(\alpha, \mathrm{n})$ sources such as ${ }^{241} \mathrm{Am}-\mathrm{Be}$, and photo-neutron sources such as ${ }^{124} \mathrm{Sb}-\mathrm{Be}$. For the third type, photo-neutron cross section is of the order millibarns, implying the need for a rather strong driving gamma source. Due to low background considerations, this option was rejected early on.

A ${ }^{252} \mathrm{Cf}$ source emits multiple neutrons per fission together with gammas. Typical $(\alpha, n)$ sources have correlated gamma neutron emission when the final state nucleus is in an excited state. As mentioned earlier, such sources would inevitably lead to correlated background in the $\mathrm{AD}$, in addition to the accidental background. Just to set the scale, the predicted correlated background for ${ }^{252} \mathrm{Cf}$ and ${ }^{241} \mathrm{Am}$-Be sources are 2.6/day and 1.3/day, respectively, assuming a $0.5 \mathrm{~Hz}$ neutron rate. There are also time-coincident 59.5 $\mathrm{keV}$ x-rays and $123 \mathrm{keV}$ gammas from ${ }^{241} \mathrm{Am}$, but their energies are too low to be detected by Daya Bay ADs.

The alphas from ${ }^{241} \mathrm{Am}$ are $\sim 5.5 \mathrm{MeV}$. To eliminate correlated gamma rays emission, ${ }^{7} \mathrm{Li}$ would be a good candidate target since such alpha energy can only produce ground state ${ }^{10} \mathrm{~B}$. However, the most common and chemically inert $\mathrm{Li}$ compound is $\mathrm{LiF}$, and $(\alpha, \mathrm{n})$ on ${ }^{19} \mathrm{~F}$ creates a significant amount of high energy gamma rays.

${ }^{13} \mathrm{C}$ is the final candidate. We note that ${ }^{241} \mathrm{Am}-{ }^{13} \mathrm{C}$ can produce neutrons with the final state ${ }^{16} \mathrm{O}$ either in the ground state, and the first or second excited state. The first excited state of ${ }^{16} \mathrm{O}$ decays into a $\mathrm{e}^{+} \mathrm{e}^{-}$pair, which 
will be stopped by the source enclosure and surrounding materials ${ }^{1}$. The second excited state of ${ }^{16} \mathrm{O}$ will emit a $6.13 \mathrm{MeV}$ gamma ray, producing correlated background together with the neutron. However if the energy of $\alpha$ is attenuated to below $5.11 \mathrm{MeV}$, this correlated $\gamma$-neutron process can be eliminated entirely. Based on all considerations above, we selected ${ }^{241} \mathrm{Am}-{ }^{13} \mathrm{C}$ as the neutron source, further requiring that $E_{\alpha}<5.11 \mathrm{MeV}$.

\section{Physical Design}

\section{1. ${ }^{241}$ Am Sources}

${ }^{241} \mathrm{Am}$ discs from NRD Inc., 5-mm in diameter, were procured with 4.5 $\mathrm{MeV}$ alpha energy as a key specification (custom energy was achieved by varying the thickness of the electrodeposited gold coating). The activity of ${ }^{241} \mathrm{Am}$ is approximately $28 \mu \mathrm{Ci}$, and is deposited on one side of the disc only. $28 \mu \mathrm{Ci}$ is the maximum activity the vendor offered to deposit for a 5$\mathrm{mm}$ disc source, and the neutron emission rate using such alpha source was estimated to be around $1 \mathrm{~Hz}$, which met our requirement. Measurements of the emitting alpha energy were performed at Caltech in a vacuum chamber with a $\mathrm{Si}$ detector. The raw energy spectrum from the ${ }^{241} \mathrm{Am}$ source is shown in Fig. 1. Also overlaid is the energy spectrum from a standard ${ }^{241} \mathrm{Am}$ source ${ }^{2}$. It was discovered that although the alpha energy for the NRD sources is peaked around $4.6 \mathrm{MeV}$, the distribution is rather broad, all the way up to $5.5 \mathrm{MeV}$. The same measurements were performed on multiple discs and the results were consistent. In order to further reduce the alpha energy, $1 \mu \mathrm{m}$ thick gold foil was purchased from Alfa Aesar and attached to the front surface of a NRD source. The attenuated energy spectrum is overlaid in Fig. 1. Compared to that without the gold foil, the entire energy spectrum was shifted by about $0.5 \mathrm{MeV}$, as expected. Out of about 20000 total alphas, no events were observed beyond $5.11 \mathrm{MeV}$ threshold.

\subsection{Expected neutron rate and energy spectrum}

A GEANT4 program was developed to calculate the neutron rate from the source. To simulate the one-sided NRD source, the alphas were generated

\footnotetext{
${ }^{1}$ The stopped $\mathrm{e}^{+}$would annihilate into two back-to-back $0.511 \mathrm{MeV}$ gammas, which can hardly deposit enough energy in the AD to cross the trigger threshold.

${ }^{2}$ A typical alpha calibration source has a thin front window of $100 \mu \mathrm{g} / \mathrm{cm}^{2}$, which attenuates the $5.5 \mathrm{MeV}$ alpha energy only by about $22 \mathrm{keV}$.
} 


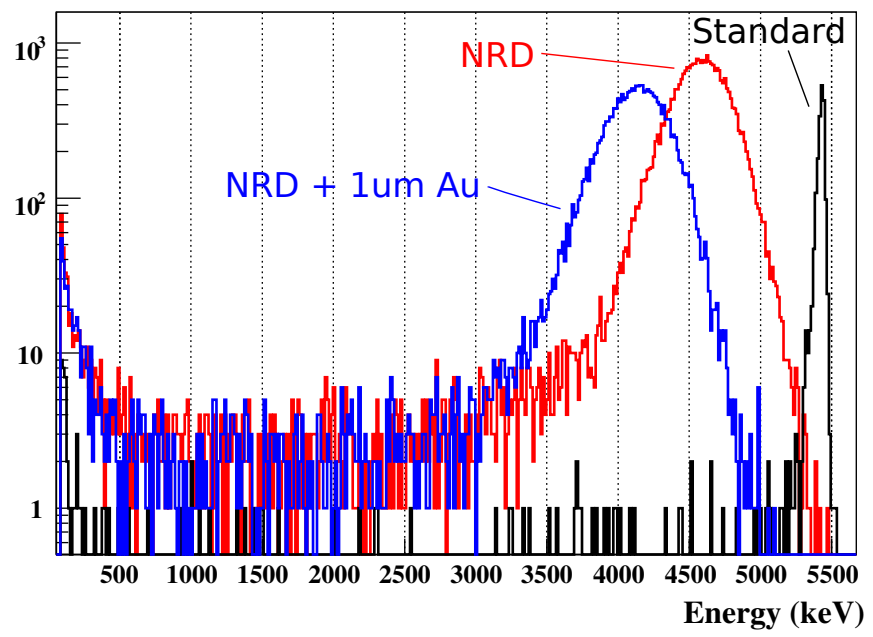

Figure 1: Measured alpha spectra with (blue) and without (red) the $1 \mu \mathrm{m}$ gold foil. The spectrum from a standard ${ }^{241} \mathrm{Am} \alpha$ source (5.5MeV) (black) is overlaid for reference.

in random directions in the active hemisphere with energy sampled from the spectrum in Fig. 1 (the red histogram). The energy loss of the alphas in $1 \mu \mathrm{m}$ gold and ${ }^{13} \mathrm{C}$ was simulated by GEANT4. GEANT4 tracks alphas until they are stopped. When an alpha enters ${ }^{13} \mathrm{C}$, for each step $i$ along the track, one computes a step weight (which gets summed at the end of the event)

$$
\text { weight }_{\mathrm{i}}=\sigma\left(E_{\alpha, i}\right) \times d_{i},
$$

where $E_{\alpha, i}$ is the mean alpha energy in this step, $d_{i}$ is the step length and $\sigma\left(E_{\alpha, i}\right)$ is the ${ }^{13} \mathrm{C}(\alpha, \mathrm{n})$ reaction cross section. The total neutron rate can then be calculated as

$$
R_{n}=R_{\alpha} \times \sum_{i} \text { weight }_{\mathrm{i}} \times \frac{\rho_{13} C_{\mathcal{N}}}{13}
$$

where $R_{\alpha}$ is the alpha emission rate, $\rho^{13} \mathrm{C}$ is the density of ${ }^{13} \mathrm{C}$, and $\mathcal{N}_{\mathcal{A}}$ is the Avogadro's number. The outgoing neutron energy spectrum was obtained by generating a neutron in random direction (but not tracked) with respect to the alpha momentum in each tracking step in ${ }^{13} \mathrm{C}$, with its energy calculated based on 2-body elastic scattering kinematics. Each neutron was assigned a step weight as in Eqn. 1. The resulting neutron energy spectrum is shown in Fig. 2(a). One sees that on average the neutron energy is $4 \mathrm{MeV}$, with a tail 
extending to $6.5 \mathrm{MeV}$ or so. The neutron energy-angle (where $\theta$ is the angle relative to the normal of the active alpha surface) correlated distribution is shown in Fig. 2(b). Clearly, neutrons heading opposite to the "active" side of the alpha source have significantly lower energy.

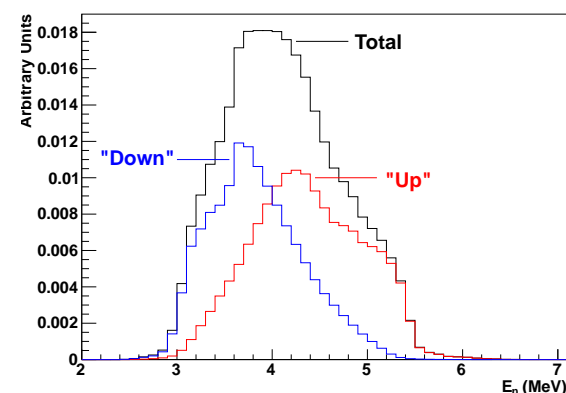

(a) Energy spectrum

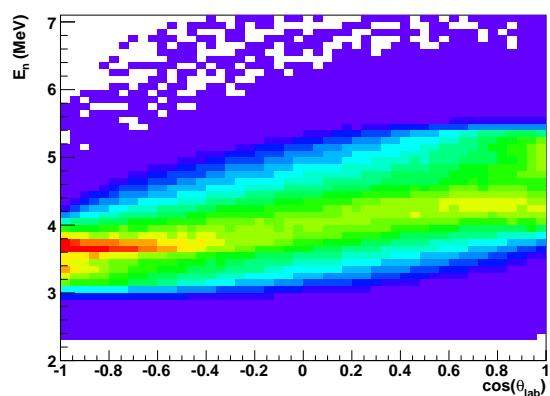

(b) Energy-angle correlation

Figure 2: a) Simulated neutron energy spectrum from the ${ }^{241} \mathrm{Am}-{ }^{13} \mathrm{C}$ source: black=total, red=heading out from the active side of ${ }^{241} \mathrm{Am}$ ("up"), blue=heading opposite to the active side of ${ }^{241} \mathrm{Am}$ ("down"). The fraction of neutrons with energy $>4.4 \mathrm{MeV}$ is $33 \%, 50 \%$, and $16 \%$ for "total", "up", and "down" distributions, respectively. b) Simulated neutron energy vs. angle distribution. See text for details.

High energy neutrons could inelastically scatter with materials surrounding and inside the detector, e.g. stainless steel, ${ }^{12} \mathrm{C}$, etc, and produce gamma rays along the way, introducing correlated background when being detected in delayed coincidence with the final SS-capture gammas. To reduce such a residual background, we chose to use the source with the active side of the ${ }^{241} \mathrm{Am}$ facing up to reduce the energy of the downward going neutrons.

\section{Mechanical Design and Fabrication}

Pure ${ }^{13} \mathrm{C}$ is immediately available in powder form. To get a good neutron flux, it is important to have the ${ }^{241} \mathrm{Am}$ in close and uniform contact with ${ }^{13} \mathrm{C}$. Alpha leakage is a serious contamination, so such a source should also be very safely sealed.

The mechanical design ${ }^{3}$ of the ${ }^{241} \mathrm{Am}-{ }^{13} \mathrm{C}$ source is shown in Fig. 3. A

\footnotetext{
${ }^{3}$ A switchable source design [5] was considered to further reduce background, but it was given up due to the complexity in automating the switch.
} 


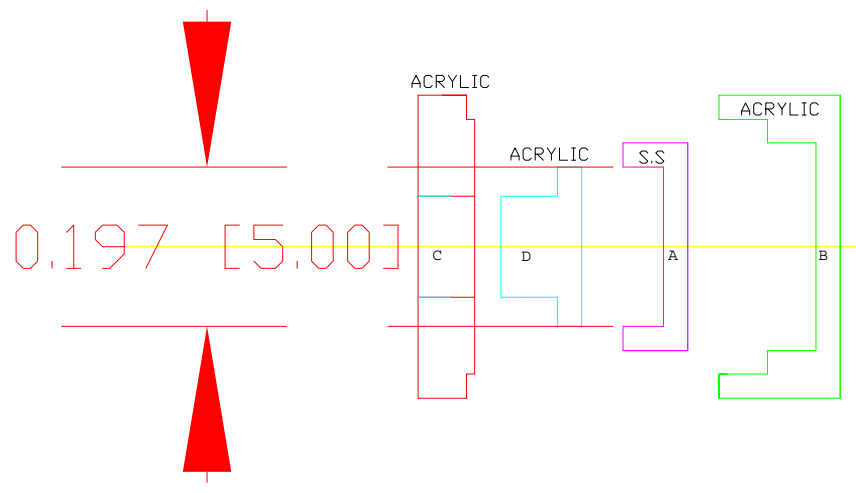

Figure 3: Mechanical drawing of the ${ }^{241} \mathrm{Am}-{ }^{13} \mathrm{C}$ source assembly. See text for more details.

stainless cup with $5 \mathrm{~mm}$ inner diameter is the holder for ${ }^{13} \mathrm{C}$, which is pressed into a solid form. ${ }^{241} \mathrm{Am}$ source, $\mathrm{Au}$ foil, and ${ }^{13} \mathrm{C}$ are sandwiched tightly. The stainless cup will be enclosed in an acrylic enclosure made out of three pieces: the bottom cup which houses the stainless cup; a plunger that presses ${ }^{241} \mathrm{Am}$, the $\mathrm{Au}$ foil, and ${ }^{13} \mathrm{C}$ together, and the top flange that seals the entire assembly. The seals between the acrylic pieces are made using the Weld-on 3 (volatile, water thin) acrylic cement [6]. Such a design went through a standard test procedure and obtained the State of California Certificate of Sealed Sources, which was a key requirement to transport these sources to Daya Bay.

\section{Quality controls}

Twenty-eight ${ }^{241} \mathrm{Am}-{ }^{13} \mathrm{C}$ sources were fabricated at Caltech, twenty-four of which were transported to Daya Bay after a quality control (QC) process. During QC, we set up a neutron detector in a low background environment, and measured the neutron rates from these sources. The detector assembly consists of an array of $4 \mathrm{NaI}$ detectors $(15 \times 15 \times 30 \mathrm{~cm})$, and neutrons are detected via

$$
{ }^{127} I+n \rightarrow{ }^{128} I+\gamma(\sim 6 \mathrm{MeV})
$$

Schematic views of the setup are shown in Fig. 4. Due to the relatively low neutron emission rate in our ${ }^{241} \mathrm{Am}-{ }^{13} \mathrm{C}$ sources, external background had to be significantly reduced to achieve a tolerable signal to background ratio. We placed a detector into a subbasement lab on Caltech campus, with an overburden of about 6 meters consisting of 1 meter of concrete and 5 meters 


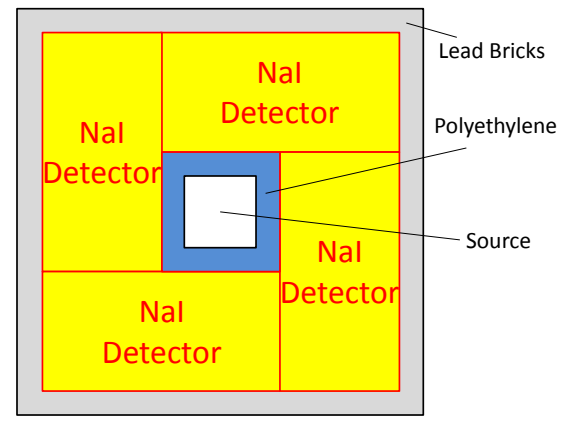

(a) Top view

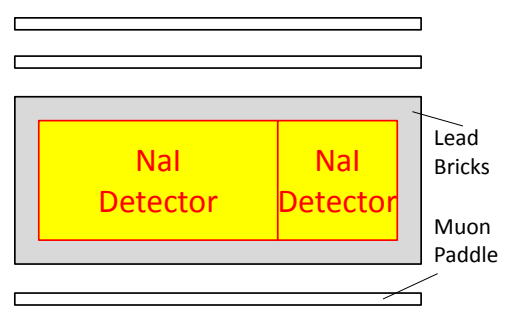

(b) Side view

Figure 4: Schematic views of the neutron detector setup.

of dirt to shield against cosmogenic neutron background. The assembly was shielded against ambient gamma rays by lead bricks of about 2 inch (5.08 $\mathrm{cm})$ thick. Two scintillator paddles $(\sim 60 \mathrm{~cm} \times 60 \mathrm{~cm} \times 2.5 \mathrm{~cm})$ were placed on the top and one on the bottom of the assembly to serve as a muon veto with an efficiency measured to be $\sim 95 \%$. The ${ }^{241} \mathrm{Am}_{-}{ }^{13} \mathrm{C}$ neutron source to be assayed was placed at the center of the detector assembly to maximize the acceptance. The threshold of each $\mathrm{NaI}$ was set at $\sim 3 \mathrm{MeV}$. Any overthreshold hit in a NaI generated a first level trigger which, in combination with the veto signals from the muon paddles ( $30 \mu$ s window), formed the main trigger to read out the ADCs/TDCs. The residual background neutron-like rate, summed over all four NaI detectors, is about $1.4(0.25) \mathrm{Hz}$ without (with) the muon veto enforced.

The neutron detection efficiency was calibrated with a standard $2.7 \mathrm{kHz}$ ${ }^{252} \mathrm{Cf}$ neutron source. The energy spectrum of the capture gammas is shown in Fig. 5. One clearly observes the iodine capture gamma "bump". When setting an energy cut between 4.5 and $8 \mathrm{MeV}$, the neutron detection efficiency is calibrated to be $5 \pm 1 \%$, where the uncertainty is dominated by the absolute neutron rate. For our weak ${ }^{241} \mathrm{Am}^{-13} \mathrm{C}$ sources, a measurement cycle consists of a 24-hour overnight background counting run, followed by a 24-hour overnight "signal" run. Statistical subtraction was made to extract the neutron source signals. The shape of the extracted neutron signals is roughly consistent with that from the calibrated sources in Fig. 5. The measured neutron rates of all 24 neutron sources are summarized in Fig. 6. The average neutron rate per source is $0.56 \mathrm{~Hz}$, with a maximum variation 


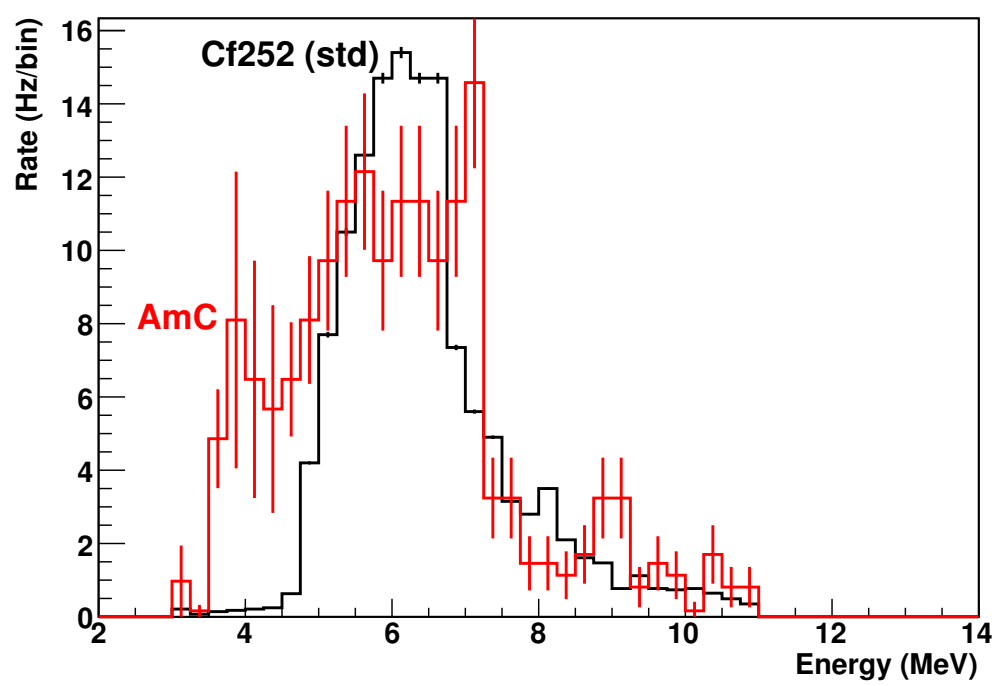

Figure 5: Detected energy spectra for neutron sources with background subtracted. Black: spectrum from standard ${ }^{252} \mathrm{Cf}$, Red: a typical ${ }^{241} \mathrm{Am}^{13} \mathrm{C}$ source, scaled for visual clarity.

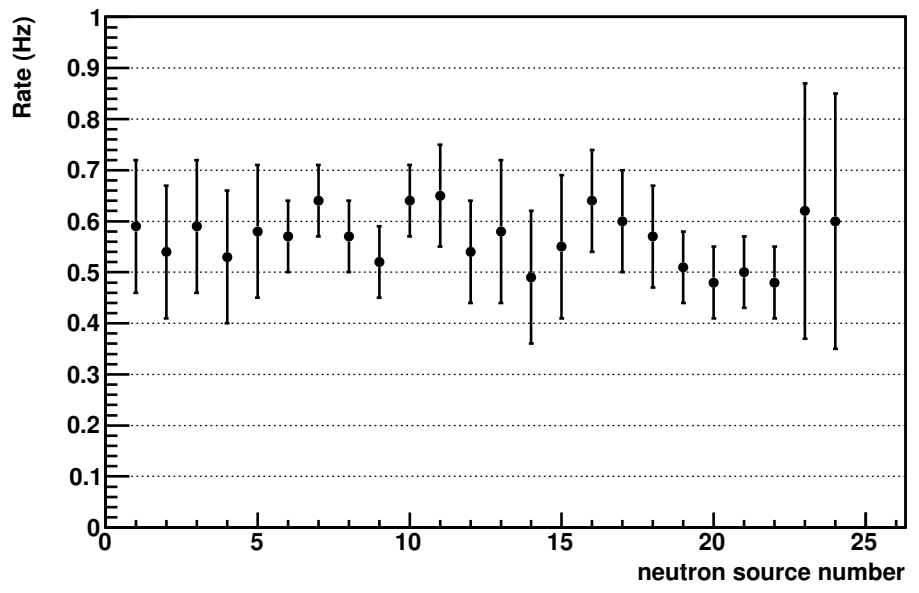

Figure 6: Summary of the rate of the neutron sources measured on the bench. Only statistical errors are shown. The larger error bars of the last two points were due to an increase of background. 
of $\pm 15 \%$ from source to source and an overall systematic uncertainty of $20 \%$ due to detection efficiency. This clearly satisfies our low rate requirement highlighted in Sec. 2.

\section{Performance of the neutron sources at Daya Bay}

Neutron sources were shipped to Daya Bay and installed in the ACUs during the detector assembly (detailed in [7]). To achieve a minimum background, at the parking location inside the ACU the neutron source is surrounded by a borated polyethylene cylinder (BPE) with 5 inch height and 2.25 inch wall thickness. Below the cylinder, there is another BPE disk with 3.25 inch in diameter and 2.5 inch in thickness acting as further shield.

Neutron sources are used extensively in calibrating the detector response, which has been reported in $[9,10]$. We limit the discussions here to some basic performance of these sources. When the sources are deployed into the detector, the proton recoil and the following capture gammas form time-correlated pairs, similar to IBD signals. The average neutron rate as measured in the detector is $0.7 \mathrm{~Hz}$, consistent with the $\mathrm{QC}$ measurements at Caltech. In Fig. 7, the prompt-delayed energy spectrum, as well as the time separation in between for an ${ }^{241} \mathrm{Am}-{ }^{13} \mathrm{C}$ source deployed in an $\mathrm{AD}$ is shown, overlaid with the expected distribution from MC. All distributions agree well with MC expectations. The single count at around $6.6 \mathrm{MeV}$ in Fig. 7(a) is consistent with our expectation of IBD signals (even if we assume it is due to the second excited state of ${ }^{16} \mathrm{O}$, it would translate into an extra background that is totally negligible). Therefore we observe no evidence of the $6.1 \mathrm{MeV}$ gamma emission, confirming that the key design specification is met. The residual background introduced by these neutrons is quite small [4]. The details of the evaluation will be discussed in a separate article [11]. 


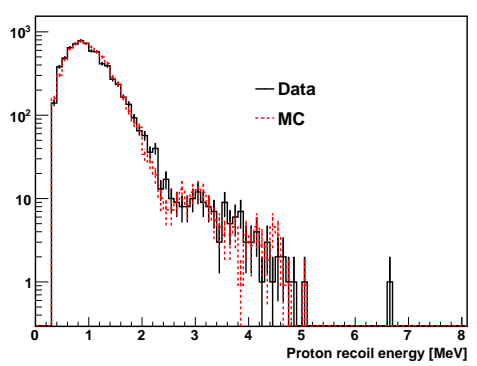

(a) Prompt energy spectrum

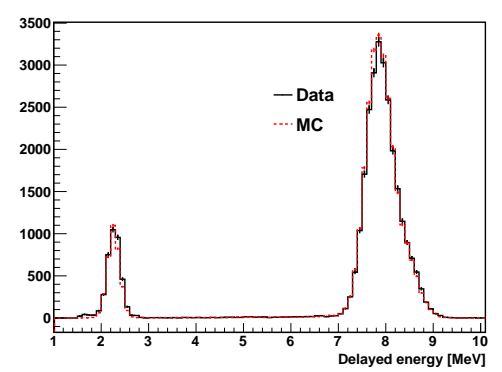

(b) Delayed energy spectrum

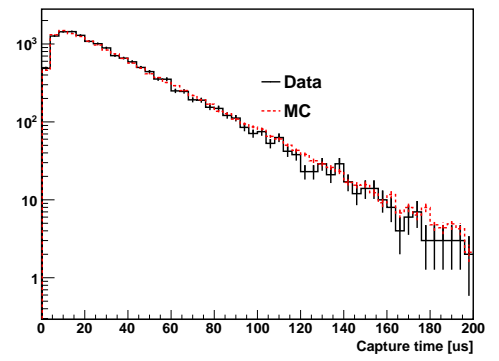

(c) Prompt-delay time separation

Figure 7: Reconstructed prompt (a) and delayed (b) energy spectra, as well as the time separation between the two (c). The data and $M C$ simulation are normalized to equal area and overlaid. 


\section{Summary}

We have discussed the design and construction of the special compact and low rate neutron sources in the Daya Bay experiment. The performance of the low background neutron sources has been proven to satisfy the design specifications at Daya Bay. Such sources could be used in other experiments requiring low backgrounds.

\section{Acknowledgments}

This work was done with support from the US DoE, Office of Science, High Energy Physics, the US National Science Foundation, the Natural Science Foundation of China Grants 11175116, the Chinese MOST grant 2013CB834306, and Shanghai Laboratory for Particle Physics and Cosmology at the Shanghai Jiao Tong University. This work is supported in part by the CAS Center for Excellence in Particle Physics (CCEPP).

We gratefully thank Dick Hahn from BNL for his critical guidance in making these sources. We also thank David Jaffe from BNL for his intellectual input. The technical support from the Kellogg technical team Ray Cortez and Jim Pendlay, particularly Ray's design work, is truly indispensable. We also appreciate the safety guidance from the Caltech radiation safety officers Haick Issaian and Andre Jefferson, and on-site logistical support from Xiaonan Li of IHEP.

[1] X. H. Guo et al. (Daya Bay Collaboration), arXiv:hep-ex/0701029.

[2] F. Ardellier et al. (Double Chooz Collaboration), arXiv:hep-ex/0606025.

[3] J. K. Ahn et al. (RENO Collaboration), arXiv:1003.1391

[4] F. P. An et al. (Daya Bay Collaboration), Phys. Rev. Lett. 108, 171803 (2012).

[5] K. H. Ansell and E. G. Hall, Neutron Sources and Applications, Proceedings of the American Nuclear Society National Topical Meeting, CONF-710402, II-90-99 (1971).

[6] http://www.weldon.com.

[7] J. Liu et al, Nucl. Instrum. Meth. A750, 19-37 (2014). 
242 [8] S. Agostineli et al. (GEANT4 Collaboration), Nucl. Instrum. Meth. A $243 \quad 506,250-303(2003)$

244 [9] F. P. An et al. (Daya Bay Collaboration), Nucl. Instrum. Meth. A 685, $245 \quad 78-97(2012)$

246

[10] F. P. An et al. (Daya Bay Collaboration), Chin. Phys. C 37, 011001

247 (2013)

248 [11] W. Q. Gu et al., in preparation. 